\title{
TIME-RESOLVED SPECTROSCOPY OF POLYMETHINE J-AGGREGATES
}

\author{
K.-H. FELLER
}

Chemical Department, Center of Optical Spectroscopy, Friedrich-Schiller-University Jena, DDR-6900 Jena, GDR

\author{
R. GADONAS, V. KRASAUSKAS
}

Laser Research Center, Vilnius State University, 232054 Vilnius, USSR

\author{
V. FIDLER, S. VAJDA \\ Chemical Faculty, Department of Physical Chemistry, Charles-University, \\ Prague, CS-12116 Prague, Albertov, CSSR
}

(Received 16 February, 1990; in final version 9 March, 1990)

The deactivation process of J-aggregates of DDEOCI (3,3'-diethyl-5,5'-diphenyl-9-ethyl-oxacarbocyanine iodide) was investigated by means of steady-state and time-resolved absorption and fluorescence spectroscopy. The decay kinetics as well as the transient absorption spectra have been analyzed including different aggregate structures and exciton-exciton annihilation.

KEY WORDS: Polymethines, aggregates, picosecond absorption spectroscopy, single photon counting, exciton-exciton annihilation.

\section{INTRODUCTION}

The photophysical properties of aggregated chromophores are of interest for several reasons. Several biologically active pigment systems, especially in photosynthesis, form dimers and higher aggregates or behave in a similar manner to dimers and aggregates. The fast energy and electron transfer to and from adsorbed dye aggregates plays a dominant role in spectral sensitization. The strong aggregation of polymethine dyes is well-known since Scheibe in 1937 discovered a new absorption band of pseudoisocyanine (PIC) in aqueous solution which he interpreted as aggregate band ${ }^{1-3}$. Typical changes in the absorption spectra due to the aggregation phenomena are depicted in Figure 1. In dependence on the concentration a blue-shifted new absorption band of the dimer and higher oligomer states (or the so-called $\mathrm{H}$-aggregate) and a red-shifted absorption band of the so-called Jaggregate ('Jelley aggregate'4) appears. There are cases where only the dimerization $^{5,6}$ or nearly only $\mathrm{J}$-aggregation ${ }^{7-8}$ was found. In general due to the instability of aggregates in solution and the low fluorescence quantum yield of typically dye 


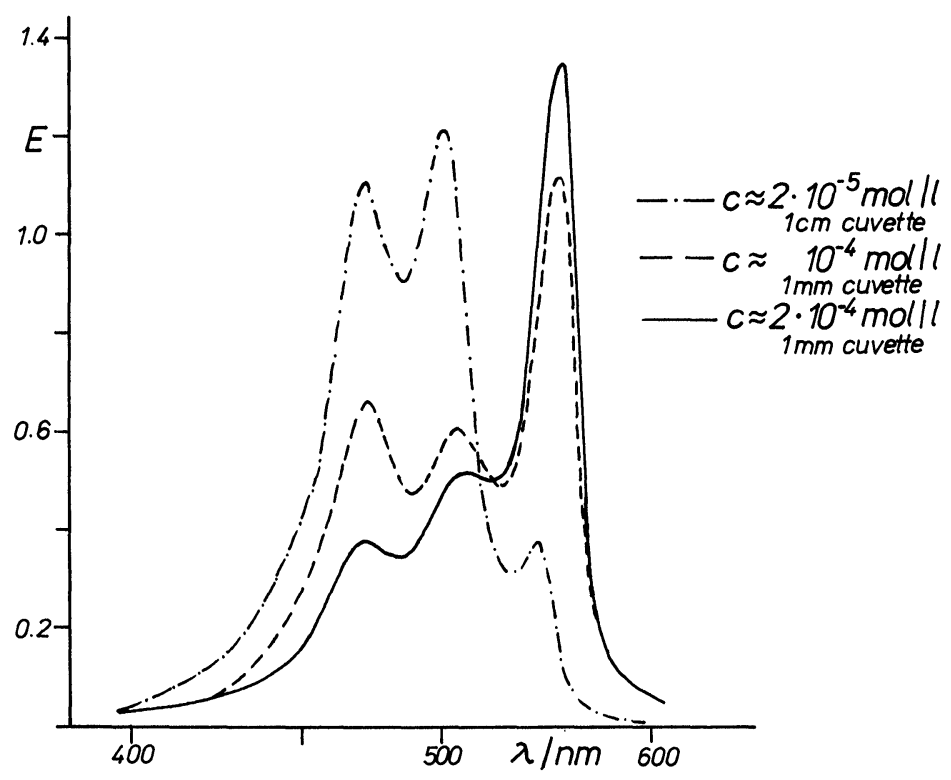

Figure 1 Absorption spectra of DDEOCI in dependence on the concentration

dimers there exist only a few time-resolved investigations of dye dimer and aggregate relaxation $^{5-10}$ (for review see ${ }^{11}$ ). The best investigated dye in this respect is the pseudoisocyanine chloride (PIC) the results of which have model character for the behavior of similar aggregates in biological molecules as chlorophyll, bacteriochlorophyll etc. Though PIC-aggregates have been investigated by several groups the results remain controverse up to now. Double-exponential decay was observed in most cases with a fast component of ca. $20 \mathrm{ps}\left(25,4 \mathrm{ps}^{12}, 21.4 \mathrm{ps}^{13}\right)$ and a slow component of ca. $600 \mathrm{ps}\left(448.3 \mathrm{ps}^{13}, \approx 600 \mathrm{ps}^{12}, 659 \mathrm{ps}^{14}\right)$. The slow component is interpreted as the lifetime of the excited aggregate molecule, whereas the fast component is due to quenching of the excitation by exciton-exciton-annihilation of the excited $\mathrm{J}$-aggregate. It was shown by $\mathrm{y}^{7,8}$ that this process is very effective at excitation intensities above $\approx 10^{19} \mathrm{phot} / \mathrm{cm}^{2} \mathrm{~s}$ or $10^{10} \mathrm{phot} / \mathrm{cm}^{2} \mathrm{pulse}$. In other cases at very high excitation intensities $\left(>10^{26} \mathrm{phot} / \mathrm{cm}^{2} \mathrm{~s}\right)$ the fast component has been detected only.

By means of nonlinear absorption investigations Stiel et al. ${ }^{7}$ have detected an intensity dependent decrease of the $S_{1}$-state lifetime above a threshold of $10^{19}$ photons $/ \mathrm{cm}^{2}$, which they interpret as an exciton-exciton annihilation process of the same manner as in chlorophyll aggregates. Sundstróm et al. ${ }^{8}$ have observed a single exponential lifetime of $400 \mathrm{ps}$ even at very low excitation intensities. At intermediate intensities the decay kinetics is strongly nonexponential. The observed intensity dependence of the decay kinetics is attributed to efficient exciton-excitonannihilation between the highly mobile singlet excitons.

The molecular mechanism responsible for these effects is explained as an interaction between the transition dipole moments of the molecules involved in the 
process. In principle, higher multipoles could also be involved. In accordance with the exciton theory two limiting cases are distinguished depending on the strength of interaction $\left(\mathrm{se}^{15}\right)$. Strong interaction is characterized by the formation of new bands in the absorption spectrum of the molecules, whereas weak interaction leads to minor distortion of the vibronic structure only. According to the Franck-Condon principle for the absorption and fluorescence processes, these characteristics may be explained in terms of fast excitation transfer among molecules in the aggregate by the fact that they do not have enough time to distort before the excitation moves to other sites. In the molecular exciton theory the strong and weak coupling concept has been used by Simpson and Peterson ${ }^{16}$ to describe the coupling strength of the excitation transfer. Briggs and Herzenberg ${ }^{17}$ and $\mathrm{Sumi}^{18}$ used coherent potential approximation to explain the absorption band shape in terms of the exciton band width $\mathrm{B}$ and the electron phonon coupling parameter S. Following these authors the band may appear when B > S $h \omega$ where $\omega$ is the characteristic frequency of the coupled molecular vibration. Hence the $\mathrm{J}$ band should appear when $\mathrm{B}$ is appreciably larger than the molecular vibrational energies ${ }^{15}$.

Contrary to the usual interpretation of the J-band by an in-phase excitation of transition dipoles along the chain (giant dipole), Dinter ${ }^{19,20}$ explained the J-band from bound states due to strong exciton-phonon interaction. These polaronic states are known as self-localized states in chain molecules of finite lengths.

$\mathrm{May}^{30}$ has proposed a three level model to account for nonlinear light absorption in the vicinity of the $\mathrm{S}_{1}$-exciton absorption of chlorophyll a. This three level model comprises the $S_{1}$-exciton as the first excited state and two $S_{1}$-excitons formed at higher light intensities as the second excited state.

The problem of the length of $\mathrm{J}$-aggregates which is controversely discussed in the literature ${ }^{7,8}$ was studied by Dinter ${ }^{19,20}$ for the case of PIC. He found that the average length of PIC J-aggregates must be less than 20 monomer units.

In a theoretical study of Scherer et al. ${ }^{21,22}$ which uses a model of Frenkel excitons interacting with intramolecular phonons the minimum number of monomeric molecules in the exciton domain of the PIC J-aggregate was found to be 6 .

The recent results of aggregation number estimations in PIC and related dye molecules from experimental data are still controverse. Stiel $e t$ al. ${ }^{7}$ have estimated from their nonlinear absorption investigations that the spectroscopical active centers of PIC J-aggregates consist of relative few molecules $(\mathrm{N}<10)$. On the other hand the domain size obtained from annihilation curves was estimated by Sundström $e t$ $a l .{ }^{8}$ to 20000 . . 50000 molecules. However, the aggregate size calculated from the nonlinear absorption investigations taking into account the smallest spectroscopically active domain and the aggregate size over which excitons can migrate freely do not necessarily need to be identical. For our investigations we used DDEOCI which shows a very strong aggregation in methanol-water mixtures. First aggregates can already be detected at dye concentrations of $10^{-6} \mathrm{~mol} / \mathrm{l}$. Whereas in pseudoisocyanine the J-aggregate band is uniform and the detected lifetime(s) belong(s) to only one aggregate structure already stationary investigations indicated that the situation in DDEOCI is more complex. The first indices are changes in the absorption maximum of the J-band in dependence on the age of the sample (see Figure 2). But also in 
dependence on the preparation conditions at least two different maxima (or a shoulder) have been found. Furthermore the DDEOCI J-aggregate band is less steep than this of PIC. From this point of view it should be neccessary to investigate different types of $J$-aggregate forming cyanines to get additionally information about effects determining the aggregate size. Also in this respect the extraordinary behavior of DDEOCI makes this dye very well-suited for detailed investigations of $\mathrm{J}$-aggregation process in polymethine dyes.

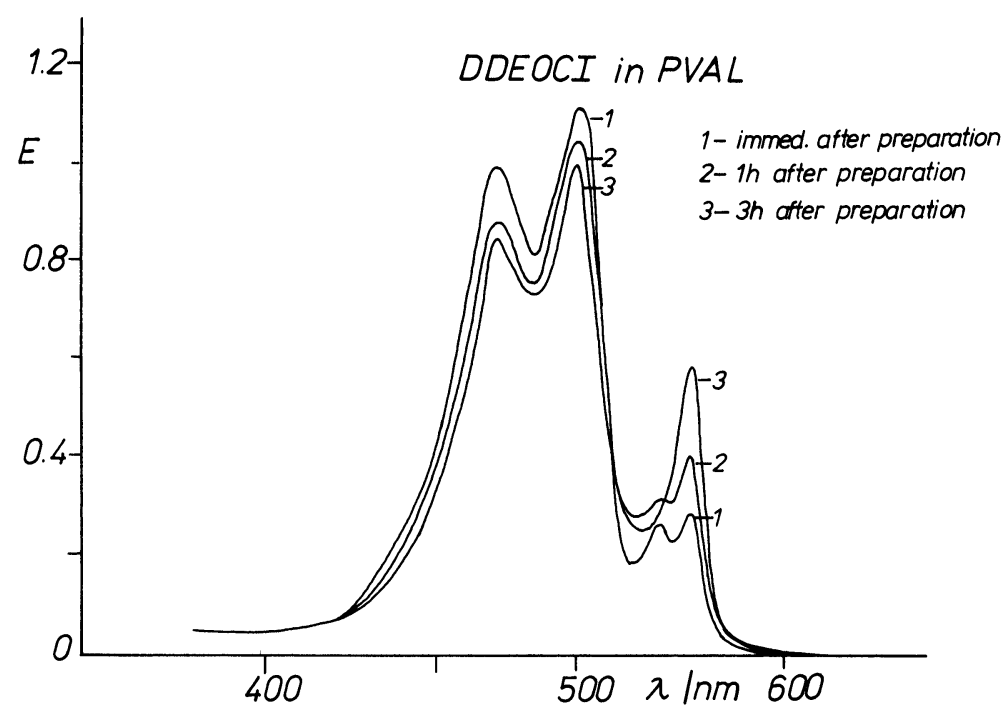

Figure 2 Changes in the absorption spectrum of DDEOCI in dependence on the age of the sample a) immediately after preparation, b) $1 \mathrm{~h}$ after preparation, c) $3 \mathrm{~h}$ after preparation.

\section{EXPERIMENTAL}

The investigated dye DDEOCI (3,3'-diethyl-5,5'-diphenyl-9-ethyl-oxacarbocyanine iodide) has been purified several times by column chromatography and by recrystallization from methanolic solution. The solvent methanol was spectroscopic grade (UVASOL) and used without further purification. The water used was bidestilled. The aggregate solutions have been prepared from a $5 \cdot 10^{-2}$ molar solution of DDEOCI in methanol by dilution 1:10 to 1:100 with water in dependence on the wished concentration and the rate between monomers, dimers and $\mathrm{J}$ aggregates. The aggregate solutions remain stable for some days. In some cases changes of the absorption maximum in dependence on the age of the sample have been detected.

Fluorescence decay measurements were done with time-correlated single-photon counting detection technique using EI 299 T (Edinburgh Instr. Ltd.) instrument. The instrument was equipped with a multiplexing electronics, allowing to do a simultaneous measurement of instrument response function on up to three fluor- 
escence decay curves. For that, T-geometry was implemented, having double SPEX monochromator in one detection channel, single monochromator with computercontrolled polarization accessory on the other detection channel (so acting effectively as two channel) and independent 'SAFE' channel (Simultaneous Acquisition of Fluorescence and Excitation) for excitation pulse profile (instrument response function) measurement. As excitation source a $\mathrm{H}_{2}$-flash lamp with a pulse width of $1.2 \ldots 1.4 \mathrm{~ns}$ FMHW was used. The excitation slit width and the slit width for the emission light were both $20 \mathrm{~nm}$. Experimental data analysis was done on PDP 11/23+ on-line computer. Mostly the software supplied by Edinbourgh Instruments was used for data analysis. Reconvolution was done on all experimental data. The goodness-of-fit was checked by fitting of the results to different numbers of exponentials by means of equation (1)

$$
\mathrm{D}=\mathrm{A} \exp \left(-\mathrm{t} / \tau_{1}\right)+\mathrm{B} \exp \left(-\mathrm{t} / \tau_{2}+\mathrm{C} \exp \left(-\mathrm{t} / \tau_{3}\right)+\ldots\right.
$$

calculating the standard deviation as well as the $\chi^{2}$-test. The channel shift technique ${ }^{23}$ was used to correct the wavelength dependence of the instrument response function.

The time-resolved absorption investigations were done with a pump-and-probe pulse spectrometer which has been described in detail elsewhere ${ }^{24}$. The samples were excited by $532 \mathrm{~nm} 25$ ps pump pulses, and the absorption spectra at different time delays as well as the kinetics at different wavelengths have been measured by means of a weak tunable probe beam. The pump pulse intensity was changed within the range $10^{10}-5 \cdot 10^{15} \mathrm{phot} / \mathrm{cm}^{2}$ per pulse by focusing or defocusing the irradiation spot.

The fitting procedure of the decay kinetics to the experimental curves in the case of the absorption investigations were done with the help of the ALAU-program package $^{25}$ taking into account multiexponential as well as nonexponential (due to exciton-exciton annihilation, see Eq. (2)) decay kinetics. In this case the goodnessof-fit was checked by calculating the standard deviation as well as the Durbin-Watson parameter.

\section{RESULTS AND DISCUSSION}

Our single-photon counting experiments have shown that the fluorescence decay of the J-aggregate band of DDEOCI can not be described by only one lifetime component even at very low excitation intensities. Both components, detected by means of SPC-investigations with subnanosecond time resolution, are in the nanosecond region. In Figure 3 a typical decay curve of DDEOCI J-aggregates detected by means of single photon counting is depicted. In the depicted case the fit leads to $3.4 \mathrm{~ns}$ and $11.7 \mathrm{~ns}$ as the life time components. All samples have been made under standard preparation conditions at various concentrations due to the age and preparation dependence of the investigations. The results at different concentrations are summarized in Table 1. No significant concentration dependence was found. The concentration was used in all cases so high, that preferentially J-aggregates and only less than $5 \%$ monomers and dimers exist in the solution. Otherwise due to the 
excitation conditions the results would be strongly influenced by rate-dependent (monomer: dimer: J-aggregate rate) changes more than by changes in the fluorescent components of the J-aggregates. Due to the well-known strong temperature dependence of the lifetime of the excited J-aggregate state ${ }^{9}$ the temperature was kept constant at $20^{\circ} \mathrm{C} \pm 0.2 \mathrm{~K}$.
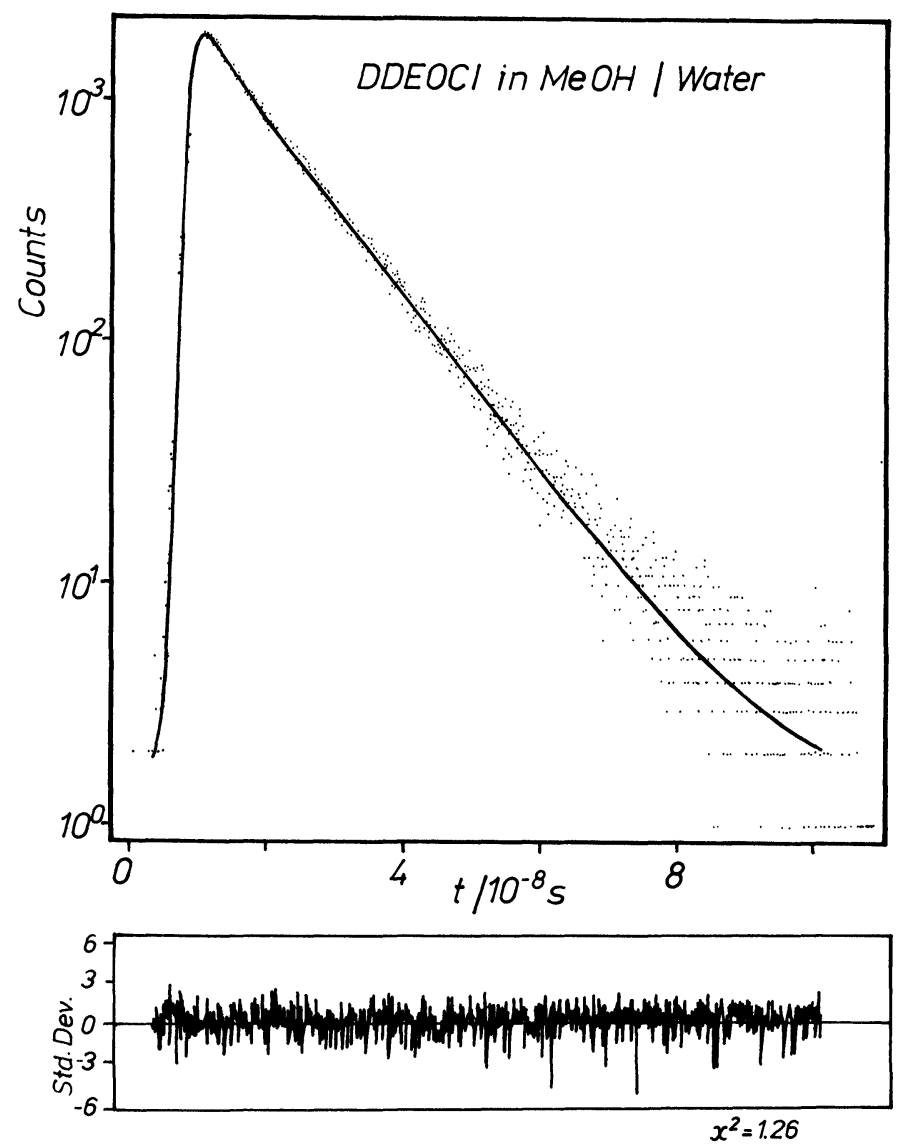

Figure 3 Detection of nanosecond decay components of DDEOCI J-aggregates by SPC-investigations

Table 1 Decay kinetics of DDEOCI J-aggregates at different concentrations (SPC measurements)

\begin{tabular}{lllllll}
\hline $\begin{array}{l}\text { concentration/ } \\
\text { l } \text { mol }^{-1}\end{array}$ & $\begin{array}{l}\text { A preexp. factor } \\
\text { to } \tau_{1}\end{array}$ & $\begin{array}{l}\tau_{1} \\
\text { Ins }\end{array}$ & $\begin{array}{l}\text { B preexp. factor } \\
\text { to } \tau_{2}\end{array}$ & $\begin{array}{l}\tau_{2} \\
\text { Ins }\end{array}$ & $\phi_{\mathrm{rel}_{1}}$ & $\Phi_{\mathrm{rel}_{2}}$ \\
\hline $1.0 \cdot 10^{-3}$ & $1.2 \cdot 10^{-2}$ & 2.9 & $2.9 \cdot 10^{-2}$ & 11.2 & $9.6 \%$ & $90.4 \%$ \\
$6.7 \cdot 10^{-4}$ & $1.7 \cdot 10^{-2}$ & 3.4 & $3.2 \cdot 10^{-2}$ & 11.7 & $13.1 \%$ & $86.9 \%$ \\
$5.5 \cdot 10^{-4}$ & $2.3 \cdot 10^{-2}$ & 3.4 & $3.2 \cdot 10^{-2}$ & 10.0 & $20.0 \%$ & $80.0 \%$ \\
$5.0 \cdot 10^{-4}$ & $9.8 \cdot 10^{-3}$ & 3.4 & $1.7 \cdot 10^{-2}$ & 10.4 & $15.5 \%$ & $84.4 \%$ \\
\hline
\end{tabular}

$$
\bar{\tau}_{1}=3.4 \mathrm{~ns} \quad \bar{\tau}_{2}=10.8 \mathrm{~ns}
$$


The existence of different (at least 2) aggregate structures is supposed to be the possible reason for the detected biexponentiality of the decay curves. As shown by means of the absorption investigations these different aggregates are furthermore able to transfer intermolecular energy between the coexisting structures.

First hints to the composition of the J-band by different types of aggregates due to different isomers have been given already by Hada et al. ${ }^{26}$ from the investigation of spectral changes of UV bands concerned with various J-aggregate types hidden in one J-band in the visible region. Furthermore in the case of 5,5'-dichlor-3,3'diethyl-9-ethyl-thiacarbocyanine chloride they found in aqueous solution three J-bands (at $619 \mathrm{~nm}$, at $623 \mathrm{~nm}$ and at $642 \mathrm{~nm}$ ). The band at $642 \mathrm{~nm}$ was shown to be unstable whereas both other bands were stable. Hada et al. ${ }^{26}$ were able to show that these spectral changes are due to different isomer-conformations in the ground state of the monomers building up different types of aggregates.

The results of the SPC investigations taken at low excitation intensity, which exclude exciton-exciton annihilation, supported the assumption of at least two different types of aggregates hidden in the aggregate band. The expected aggregate structures should coincide with detected conformer equilibrium in the ground state of meso-substituted oxacarbocyanine dyes ${ }^{27}$ where in accordance with results of Grahn and coworkers ${ }^{28}$ an equilibrium of all-trans and 8, 9 mono-cis isomers in the ground state have been found.

The results of the picosecond absorption investigations at medium excitation $\left(2 \cdot 10^{10}\right.$ phot $/ \mathrm{cm}^{2}$ per pulse) confirming the SPC-results are depicted in Figure 4. It is seen that the deactivation kinetics should be fitted to an at least triple exponential decay (see Equ. (1)) with lifetime components of

$$
\tau_{1}=26 \mathrm{ps}, \tau_{2}=295 \mathrm{ps} \text { and } \tau_{3}=4.30 \mathrm{~ns}
$$

The calculated DW-parameter $=1.62$ indicates a reasonable fit of the experimental data to the physical model. This lifetime components should be explained to belong to different aggregate structures as well as exciton-exciton annihilation.

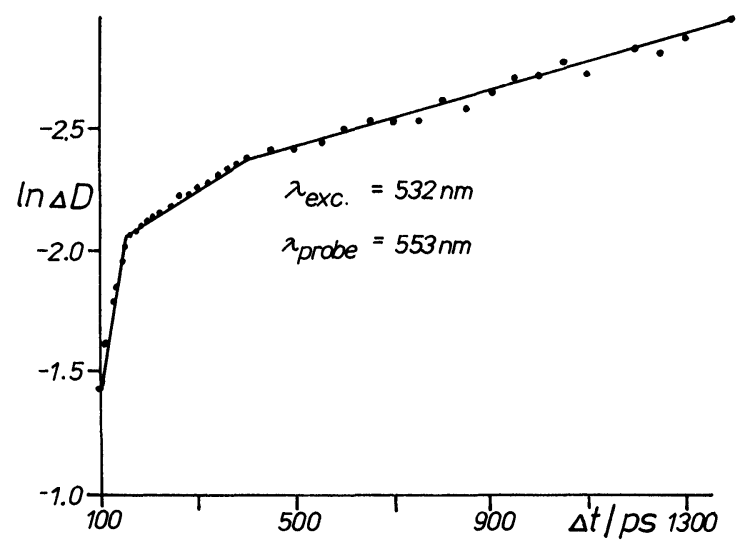

Figure 4 Detection of picosecond decay components of DDEOCI J-aggregates by pump-and-probe pulse spectroscopy 
Due to the limited delay time range of the used pump- and probe-pulse spectrometer of $1.5 \mathrm{~ns}$ longer lifetimes than some nanoseconds can not be resolved. From this point of view $\tau_{3}$ coincide with the SPC lifetimes whereas the two ps components should be connected with exciton-exciton annihilation ${ }^{11,29}$. We have checked the intensity dependence of the lifetime components to varify the excitonexciton annihilation model.

According to the exciton-exciton annihilation concept the decay kinetics should be described by the differential equation

$$
\frac{\mathrm{d} \triangle \mathrm{D}}{\mathrm{dt}}=-\mathrm{k}_{1} \triangle \mathrm{D}-\mathrm{k}_{2} \triangle \mathrm{D}^{2}
$$

of which the solution is

$$
\Delta \mathrm{D}(\mathrm{t})=\frac{2 \mathrm{k}_{2} \mathrm{~A}_{0}}{\exp \mid\left(\mathrm{k}_{1} \mathrm{t}\right)\left(\mathrm{k}_{1}+2 \mathrm{k}_{2} \mathrm{~A}_{0}\right)-2 \mathrm{k}_{2} \mathrm{~A}_{0}}
$$

describing the decay of excited aggregates by competitive exciton-exciton annihilation and first-order decay ${ }^{11}$. Whereas in the case of uniform aggregates as PIC such a fit should lead to reasonable results in our case due to the involvement of different aggregate structures and their decay kinetics we found a better fit of the decay curves by multiexponential fitting by equation (1).

Within the used excitation intensity range no significant changes in the rates and the preexponential factors of the components was seen.

The investigation of the absorption spectra of DDEOCI J-aggregates at different delay times has shown that the bleaching curve in the ground state recovery region does not vanish uniformly. There is a short-wavelength part of the bleaching curve which decays very fast whereas the long wavelength part decays very slowly (see Figure 5). As it was shown the decay kinetics of polymethine aggregates depend on the excitation intensity ${ }^{7,8}$ connected with exciton-exciton annihilation at high excitation intensities. In connection with this we have measured the influence of excitation intensity upon the decay kinetics and the transient absorption spectra. In Figure 5 and Figure 6 difference absorption spectra at high and low excitation intensities are depicted. The high excitation case is connected with an absorption at $570 \mathrm{~nm}$ with decays within 600 ps. Furthermore there is a new very weak absorption band with a maximum at $680 \mathrm{~nm}$ which is nearly constant during the available delay time changes of $1.5 \mathrm{~ns}$. In the $510 \mathrm{~nm}$ region, where the J-aggregates have a weak absorption, the bleaching of this ground state absorption is seen. In the low excitation case neither an absorption at $570 \mathrm{~nm}$ nor a weak red-shifted absorption appears.

The bleaching of the ground state absorption at $510 \mathrm{~nm}$ disappears within the used delay time of $600 \mathrm{ps}$. In both cases different sides of the J-aggregate bleaching curve do not relax uniformly. The pumping of the aggregates leads in both cases to a fast deactivation of the aggregates with blue-shifted absorption spectrum compared with the aggregates with red-shifted absorption spectrum. Even a transfer from one structure to the other one has to be taken into account. Such a transfer is indicated by kinetical measurements with excitation at $532 \mathrm{~nm}$ and probing at $568 \mathrm{~nm}$ where 


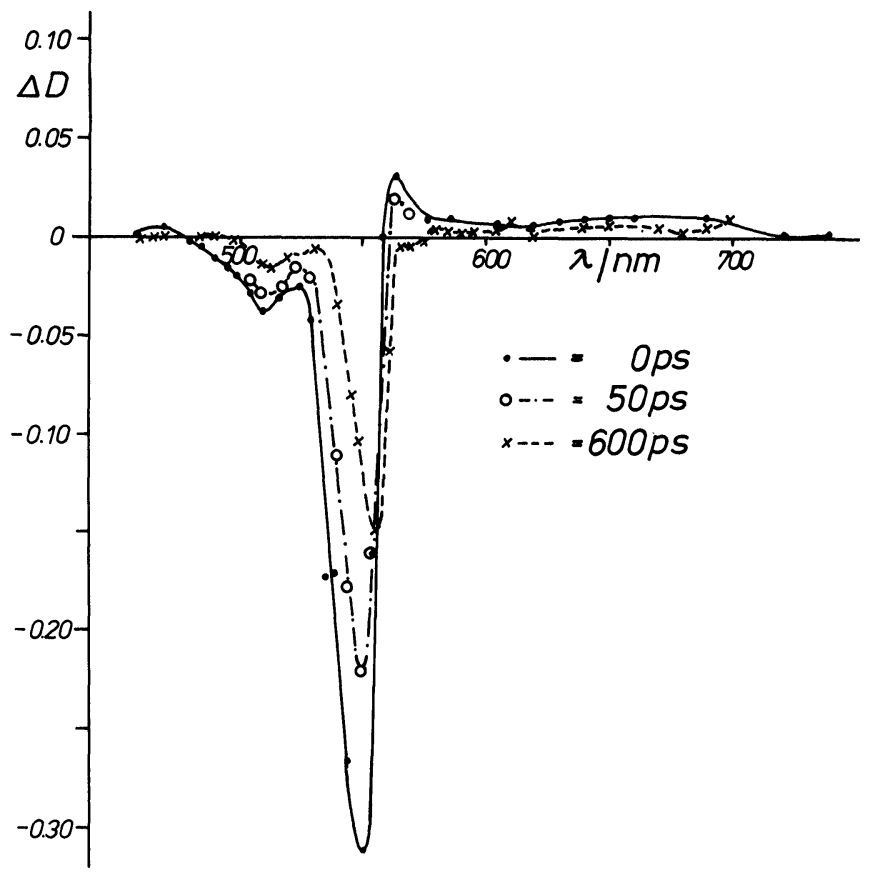

Figure 5 Picosecond absorption spectra of DDEOCI J-aggregates in water/methanol mixture at high excitation intensity excited at $532 \mathrm{~nm}$. -0 ps delay, $O-50$ ps delay, $\times-600$ ps delay.

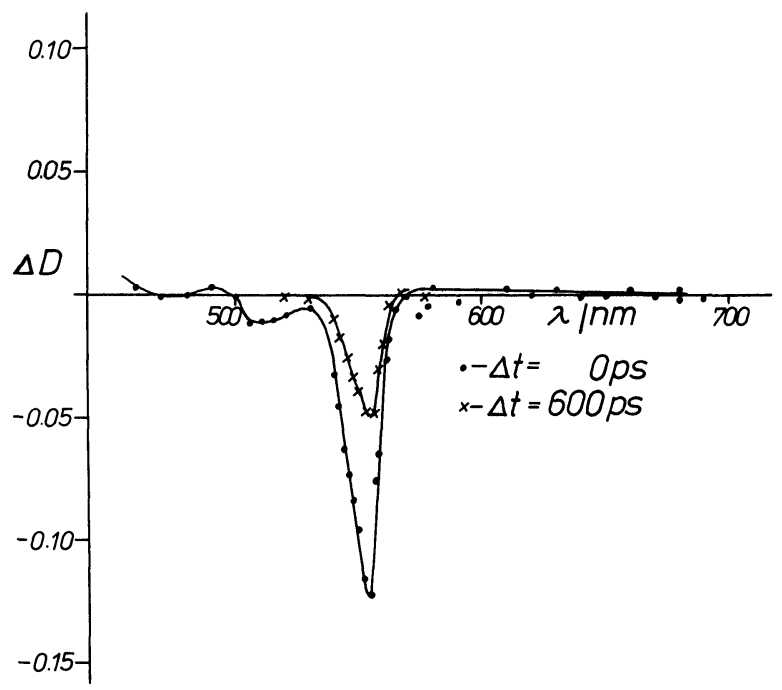

Figure 6 Picosecond absorption spectra of DDEOCI J-aggregates in water/methanol mixture at medium excitation intensity excited at $532 \mathrm{~nm},-0$ ps delay, $x-600$ ps delay. 


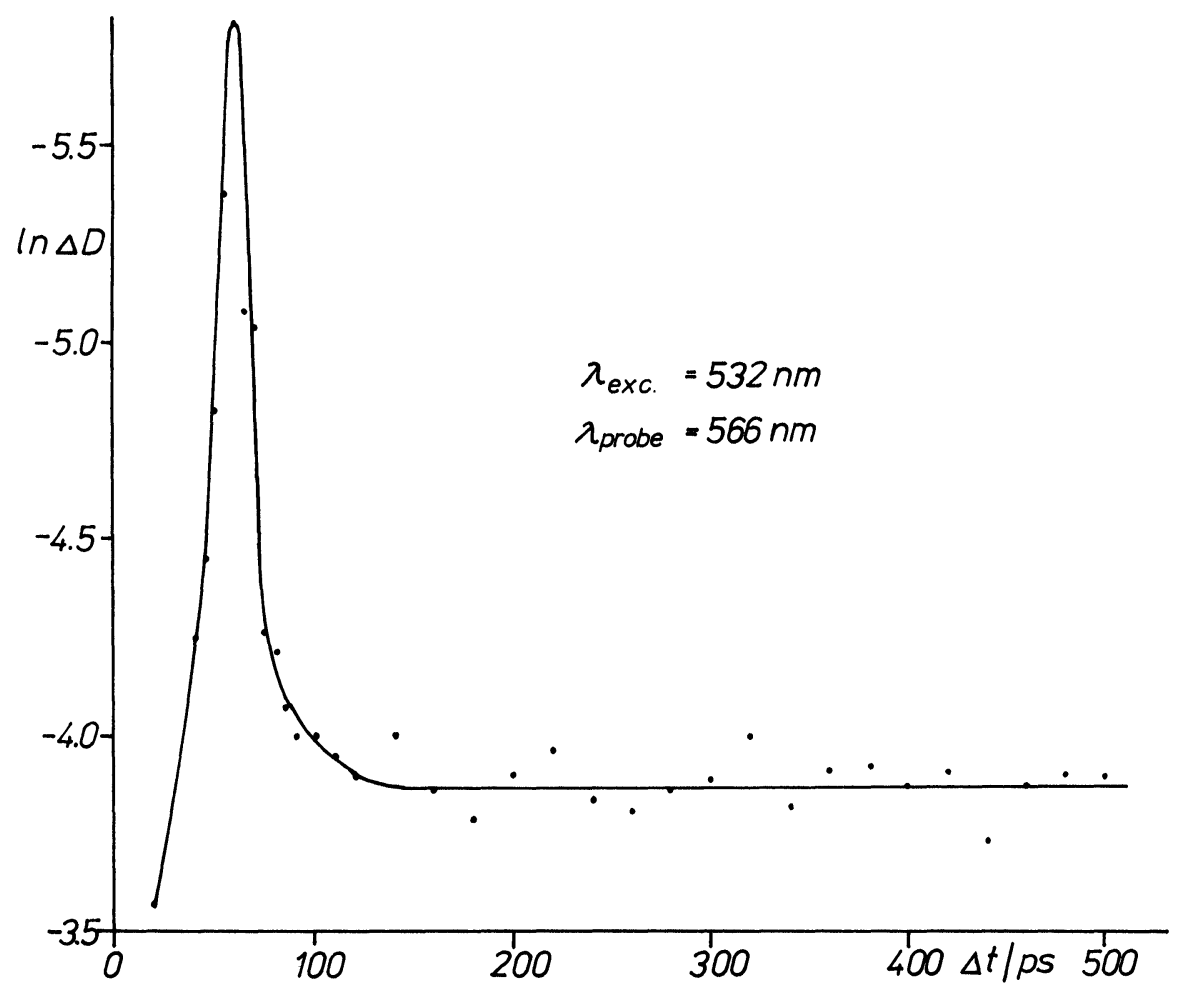

Figure 7 Decay curve of the transient absorption in the DDEOCI J-aggregate transient spectra at high excitation intensity excited at $532 \mathrm{~nm}$ and probed at $566 \mathrm{~nm}$.

additionally to the slow decay of the bleaching a fast build-up process appears at short delay times (Figure 7). This absorption band at $568 \mathrm{~nm}$ is built up within appr. $18 \mathrm{ps}$ and decays double-exponential with a similar fast first decay time of $22 \mathrm{ps}$ and a slow decay time of some nonoseconds. Excited $S_{1}$-exciton states ${ }^{30}$ could be the reason for the observed fast build-up process absorbing within the absorption band of the J-aggregate and decaying within some picoseconds to the ground state of the $\mathrm{J}$-aggregate. A small part of appr. $10 \%$ remains within the used delay times of $1.5 \mathrm{~ns}$ without observable decay in the excited- $S_{1}$-exciton state. According to $\mathrm{May}^{30}$ the population of the $S_{1}$-exciton state depends very sensitively upon the excitation intensity and the particle density. Our results show that the observed transient absorption band vanishes at excitation intensities of $<2 \cdot 10^{10} \mathrm{phot} / \mathrm{cm}^{2}$ pulse (see Figure 6).

Aggregation is also observed in nonaqueous solutions under circumstances where appropriate solvent structuring occurs ${ }^{31}$.

Also in the case of toluene solution multiexponential behavior of the decay kinetics was found. The spectral behavior of the J-aggregate band was similar to the aqueous solution one. We can conclude also from these results a superposition of 
J-aggregate bands of different aggregate structure as reason for the less steep aggregate band compared with the PIC-J-aggregate one. From this point of view some results in the literature have to be reinterpreted.

J-aggregation of cyanine dyes at Langmuir-Blodgett films has been investigated with time-resolved methods by Lehmann ${ }^{32}$ and Nakano et al. ${ }^{33}$. The latter have found two different J-bands of meso-substituted cyanine dyes in monolayer assemblies. In dependence on the surface pressure the two different J-bands can be reversibly altered. No explanation was given for the obtained surface pressure dependence. It can be supposed that also in this case different aggregate structures in dependence on the surface pressure are the reason for the observed two J-bands.

On the other hand by means of picosecond pump-and-probe spectroscopy Quitevis $\mathrm{et} \mathrm{al.}{ }^{34}$ have investigated the electronic energy relaxation in aggregates of PIC on colloidal silica. Nonexponential signals obtained from transient optical bleaching of the J-band are discussed in terms of a polariton model. Excitation intensity of $1.5 \cdot 10^{17} \mathrm{phot} / \mathrm{cm}^{2}$ pulse is connected with exciton-exciton-annihilation rather than with the distribution of $\mathrm{J}$-aggregates with different relaxation times in this case.

The spectral properties of $\mathrm{J}$-aggregates of cyanine dyes in solution and their absorption onto silver halides have been reviewed by $\mathrm{Herz}^{35}$. Also these results gave hints to include different aggregate structures arising from different isomers as explanation of their spectral properties. Work is in progress to transfer our results on the situation in the adsorbed state. Additionally to the investigation of J-aggregates of polymethine dyes we have done some work about dimer deactivation kinetics in the same system for comparison ${ }^{36}$.

\section{Acknowledgements}

The authors are deeply indebted to Prof. A. Piskarskas (Vilnius) for encouraging their work and for very helpful discussions. Valuable discussions with Prof. S. Dähne (Berlin) and Prof. D. Fassler (Jena) are gratefully acknowledged. Furthermore we thank Mrs. E. Kielmann for technical assistance.

\section{References}

1. G. Scheibe, Angew. Chemie 50, 212 (1937).

2. G. Scheibe, Angew. Chemie 52, 631 (1939).

3. G. Scheibe, Z. Elektrochem. 52, 283 (1948).

4. E. Jelley, Nature 12, 1009 (1936).

5. S. Rentsch, D. Fassler, P. Hampe, R. Danielius and R. Gadonas, Chem. Phys. Lett. 89, 249 (1982).

6. B. Kopainsky and W. Kaiser, Chem. Phys. Lett. 88, 357 (1982).

7. H. Stiel, K. Teuchner and S. Dähne, J. Lumin. 39, 351 (1988).

8. V. Sundström, T. Gillbro, R. Gadonas and A. Piskarskas, J. Chem. Phys. 89, 2754 (1988).

9. H. Stiel, K. Teuchner, W. Becker, W. Freyer and S. Dähne, J. Mol. Struct. 114, 351 (1984).

10. V. Sundström and T. Gillbro, J. Chem. Phys. 83, 2733 (1985).

11. V. Brückner, K.-H. Feller and U.-W. Grummt, Applications of Time-Resolved Optical Spectroscopy (Elsevier Science Publishers, Netherlands, 1989).

12. K. Berndt, Opt. Commun. 56, 30 (1985).

13. H. P. Dorn and A. Müller, Appl. Phys. B43, 167 (1987).

14. D. V. Brumbaugh, A. A. Muenter, W. Knox, G. Mourou and B. Wittmershaus, J. Lumin. 31 \& 32, 783 (1984). 
15. J. Tanaka, M. Tanaka and N. Kanamura, in: Relaxation of Elementary Excitation, edited by R. Kubo and E. Hanamura (Springer Verlag, Berlin (West) 1980).

16. W. T. Simpson, D. L. Peterson, J. Chem. Phys. 26, 588 (1957).

17. J. S. Briggs and A. Herzenberg, Mol. Phys. 21, 865 (1971).

18. H. Sumi, J. Phys. Soc. Jpn. 38, 825 (1975).

19. M. Dinter, J. Chem. Phys. 80, 2914 (1984).

20. M. Dinter, J. Chem. Phys. 81, 3256 (1984).

21. P. O. J. Scherer, S. F. Fischer, K. W. Knapp, Chem. Phys. Lett. 106, 191 (1984).

22. P. O. J. Scherer, S. F. Fischer, K. W. Knapp, Chem. Phys. 86, 269 (1984).

23. J. Birch and H. Imhof, Anal. Instrum. 14, 433 (1985).

24. R. Danielius, R. Gadonas and A. Piskarskas, Kvantovaya Elektronika 8, 669 (1981).

25. H. Schütz, E. Stutter, K. Geller and I. Petri, Studia Biophysica 104, 23 (1984).

26. H. Hada, C. Honda and H. Tanemura, Photogr. Sci. Eng. 21, 83 (1977).

27. K.-H. Feller, R. Gadonas and V. Krasauskas, Laser Chemistry 8, 39 (1988).

28. R. Allmann, H.-J. Anis, R. Benn, W. Grahn, S. Olejnek and A. Waskowska, Angew. Chem. Suppl. 1147 (1983).

29. R. Gadonas, R. Danielius, A. Piskarskas, T. Gillbro and V. Sundström, Proceedings UPS 87 (World Scientific Publ. Co., Singapore, 1988), p. 399.

30. V. May, private communication.

31. K. -H. Feller, J. Siegel, D. Fassler, H. Bergner and F. Kerstan in: Teubner Texte zur Physik, Vol. 4, Proceedings of the Third Symposium Optical Spectroscopy (B.S.B. B.G. Teubner Verlagsgesellschaft, Leipzig, 1985) p. 216.

32. U. Lehmann, Thin Solid Films 160, 257 (1988).

33. A. Nakano, S. Shimizu, T. Takahashi, H. Nakahara and K. Fukuda, Thin Solid Films 160, 303 (1988).

34. E. L. Quitevis, M.-L. Horng and S.-Y. Chen, J. Phys. Chem. 92, 256 (1988).

35. A. H. Herz, Adv. Colloid. \& Interface Sci. 8, 237 (1977).

36. K.-H. Feller, D. Fassler, R. Gadonas, V. Krasauskas, A. Pelakauskas and A. Piskarskas, J. Mol. Struct. (in preparation). 